\title{
STUDY OF A STOCHASTIC FAILURE MODEL IN A RANDOM ENVIRONMENT
}

\author{
JI HWAN CHA,* Pukyong National University \\ JIE MI, ${ }^{* *}$ Florida International University
}

\begin{abstract}
Most devices (systems) are operated under different environmental conditions. The failure process of a system not only depends on the intrinsic characteristics of the system itself but also on the external environmental conditions under which the system is being operated. In this paper we study a stochastic failure model in a random environment and investigate the effect of the environmental factors on the failure process of the system.
\end{abstract}

Keywords: Environmental condition; failure process; Poisson shock process; baseline failure rate function; compound failure rate function; stochastic ordering

2000 Mathematics Subject Classification: Primary 60E99

Secondary $60 \mathrm{~K} 10$

\section{Introduction}

Many of the currently used failure models have been developed on the premise that the operating environment is static. In these cases, the basic assumption is that the prevailing environmental conditions either do not change in time or, if they do, have no effect on the deterioration and failure process of the device. Therefore, in these cases it is models not depending on the external environmental conditions that have been proposed and studied.

However, devices often work in varying environments and their performance can be significantly affected by the varying environmental conditions. For example, jet engines are constantly subject to varying atmospheric conditions like pressure, temperature, and humidity, as well as shocks caused by mechanical vibrations during takeoff, cruising, and landing. As another example, some electrical devices are frequently subject to random shocks caused by the fluctuations of an unstable power supply. In these cases, the changes in external conditions or shocks cause the equipment to deteriorate or age according to certain rules, and to be effectively older than its intrinsic age. In this regard, there have been various failure models for systems in random environments. The explicit consideration of the environment as a stochastic process has been attempted in a number of papers. Most such models take the model of Esary et al. (1973) as their starting points, and model the shock and wear intensities as varying randomly in response to environmental conditions; see A-Hameed and Proschan (1973), (1975), Çinlar (1984), and Feldman (1976), (1977). These models are for one component; the use of multicomponent models was discussed by Esary and Marshall (1974), and very sophisticated models were introduced by Çinlar (1977).

Received 30 March 2005; revision received 19 November 2006.

* Postal address: Division of Mathematical Sciences, Pukyong National University, Busan, 608-737, Korea.

Email address: jhcha@pknu.ac.kr

** Postal address: Department of Statistics, Florida International University, Miami, FL 33199, USA.

Email address: mi@fiu.edu 
The intrinsic ageing model was considered by Çinlar and Özekici (1987) and studied further by Çinlar et al. (1989). In these studies, the intrinsic ageing concept was introduced to relate the deterioration of a component under field conditions to the deterioration it would experience under laboratory conditions. The concept was extended in Shaked and Shanthikumar (1989). Özekici (1995) studied optimal maintenance policies for systems in random environments and Özekici (1996) discussed the implications of the environmental process in the context of inventory models, queueing models, and reliability models. The concept of random hazard functions was used and discussed by Gaver (1963), Arjas (1981), and Kebir (1991). In Lemoine and Wenocur (1985), (1986), the item's failure rate was described using a stochastic process which represents the random system state. An excellent survey of the abundant research on failure models for systems in random environments can be found in Singpurwalla (1995).

In this paper, we study a new stochastic failure model which incorporates varying external environmental conditions characterized by a Poisson shock process. In the next section we shall derive the compound failure rate function of a system in a random environment and investigate the effect of the environmental factors on the failure process. In Section 3 we will give various examples to show that, depending on the intensity function of the Poisson shock process, the compound failure rate function can have many different shapes. In Section 4 the dependence of the compound failure rate function on the intensity function of the Poisson shock process and the increment in the failure rate will be studied. Concluding remarks are given in the last section.

\section{Main results}

Let $X$ be the lifetime of a device operated in a normal, static working environment (or a laboratory environment which is not exposed to the external environmental conditions) and denote its survival function and failure rate function as $\bar{F}_{0}(t)$ and $r_{0}(t)$, respectively. The latter is called the baseline failure rate function. Let $\{N(t): t \geq 0\}$ be a stochastic counting process describing the occurrence of certain environmental shocks in $[0, \infty)$. Denote the times of occurrences of these shocks as $T_{i}, 0=T_{0}<T_{1}<T_{2} \cdots$. Let $Y$ be the lifetime of an identical device under the influence of this environmental shock process. In this paper we assume that

$$
\mathrm{P}(Y>t \mid N(s), 0 \leq s \leq t)=\exp \left\{-\int_{0}^{t}\left[r_{0}(x)+\sum_{j=1}^{N(t)} \mu \mathbf{1}_{\left[T_{j}, \infty\right)}(x)\right] \mathrm{d} x\right\}, \quad t \geq 0,
$$

where $\mu>0$ is a constant and the indicator function $\mathbf{1}_{\left[T_{j}, \infty\right)}(x), j=1,2, \ldots$, is defined by

$$
\mathbf{1}_{\left[T_{j}, \infty\right)}(x)= \begin{cases}1 & \text { if } x \in\left[T_{j}, \infty\right) \\ 0 & \text { otherwise }\end{cases}
$$

Physically, (1) means that, given the shock process in the time interval $[0, t]$, the failure rate of $Y$ increases by a fixed amount $\mu>0$ at the occurrence of each shock. Furthermore, it is assumed that a shock does not cause immediate system failure.

For structural systems, a shock may cause immediate system failure. For example, a strong earthquake can break a bridge with some probability, or may increase its deterioration by some amount. On the other hand, for electrical systems, there may be some different cases. A power surge may be a shock that causes immediate failure. However, in most electrical systems there are built-in protective devices which prevent immediate system failure by cutting off the supply of electric power in the occurrence of a surge exceeding a prespecified level. In this case, a 
shock of this kind cannot cause immediate system failure, but it increases the possibility of system failure by increasing the failure rate by some amount.

For convenience we will refer to $\mu$ as the increment in failure rate. The following result gives the failure rate function of $Y$, namely the compound failure rate function of the system.

Theorem 1. Suppose that $\{N(t): t \geq 0\}$ is a Poisson process with intensity function $\lambda(t) \geq 0$, i.e. $m(t):=\mathrm{E}(N(t))=\int_{0}^{t} \lambda(x) \mathrm{d} x$. Assuming that (1) holds and that $m(t)$ has inverse $m^{-1}(t)$, the failure rate function of $Y$, denoted by $r(t)$, is given by

$$
r(t)=r_{0}(t)+\mu \mathrm{e}^{-\mu t} \int_{0}^{t} \mathrm{e}^{\mu x} \lambda(x) \mathrm{d} x .
$$

Proof. It is easy to see that

$$
\mu \sum_{j=1}^{N(t)} \int_{0}^{t} \mathbf{1}_{\left[T_{j}, \infty\right)}(x) \mathrm{d} x=\mu \sum_{j=1}^{N(t)}\left(t-T_{j}\right)=\mu t N(t)-\mu \sum_{j=1}^{N(t)} T_{j},
$$

since $\mathbf{1}_{\left[T_{j}, \infty\right)}(x)=0$ for $0 \leq x \leq t$ and $j=N(t)+1, N(t)+2, \ldots$ Thus,

$$
\mathrm{P}(Y>t \mid N(s), 0 \leq s \leq t)=\bar{F}_{0}(t) \exp \left\{-\mu t N(t)+\mu \sum_{j=1}^{N(t)} T_{j}\right\} .
$$

From (3) we see that

$$
\mathrm{P}(Y>t)=\bar{F}_{0}(t) \mathrm{E}\left(\exp \left\{-\mu t N(t)+\mu \sum_{j=1}^{N(t)} T_{j}\right\}\right) .
$$

Define $N^{*}(t):=N\left(m^{-1}(t)\right), t \geq 0$, and $T_{j}^{*}:=m\left(T_{j}\right), j \geq 1$. It is known that $\left\{N^{*}(t): t \geq 0\right\}$ is a stationary Poisson process with intensity 1 (see, e.g. Çinlar (1975, pp. 95-96)), and $T_{j}^{*}$, $j \geq 1$, are the times of occurrence of shocks on the new time scale. Let $s=m(t)$. By (4), we have

$$
\begin{aligned}
\mathrm{P}\left(Y>m^{-1}(s)\right) & =\bar{F}_{0}\left(m^{-1}(s)\right) \mathrm{E}\left(\exp \left\{-\mu m^{-1}(s) N\left(m^{-1}(s)\right)+\mu \sum_{j=1}^{N\left(m^{-1}(s)\right)} T_{j}\right\}\right) \\
& =\bar{F}_{0}\left(m^{-1}(s)\right) \mathrm{E}\left(\exp \left\{-\mu m^{-1}(s) N^{*}(s)+\mu \sum_{j=1}^{N^{*}(s)} T_{j}\right\}\right)
\end{aligned}
$$

Considering conditional expectation, we have

$$
\begin{aligned}
& \mathrm{E}\left(\exp \left\{-\mu m^{-1}(s) N^{*}(s)+\mu \sum_{j=1}^{N^{*}(s)} T_{j}\right\} \mid N^{*}(s)=n\right) \\
& \quad=\exp \left\{-n \mu m^{-1}(s)\right\} \mathrm{E}\left(\exp \left\{\mu \sum_{j=1}^{n} m^{-1}\left(T_{j}^{*}\right)\right\} \mid N^{*}(s)=n\right) .
\end{aligned}
$$


It is known that the joint distribution of $\left(T_{1}^{*}, T_{2}^{*}, \ldots, T_{n}^{*}\right)$ given $N^{*}(s)=n$ is the same as the joint distribution of $\left(V_{(1)}, V_{(2)}, \ldots, V_{(n)}\right)$, where the $V_{(i)}, V_{(1)} \leq V_{(2)} \leq \cdots \leq V_{(n)}$, are the order statistics of the independent, identically distributed random variables $V_{1}, V_{2}, \ldots, V_{n}$, which are uniformly distributed on the interval $[0, s]=[0, m(t)]$. Hence, in (5),

$$
\begin{aligned}
\mathrm{E}\left(\exp \left\{\mu \sum_{j=1}^{n} m^{-1}\left(T_{j}^{*}\right)\right\} \mid N^{*}(s)=n\right) & =\mathrm{E}\left(\exp \left\{\mu \sum_{j=1}^{n} m^{-1}\left(V_{(j)}\right)\right\}\right) \\
& =\mathrm{E}\left(\exp \left\{\mu \sum_{j=1}^{n} m^{-1}\left(V_{j}\right)\right\}\right) \\
& =\mathrm{E}\left(\exp \left\{\mu m^{-1}(s U)\right\}\right)^{n},
\end{aligned}
$$

where the second equality follows from $\sum_{j=1}^{n} m^{-1}\left(V_{(j)}\right)=\sum_{j=1}^{n} m^{-1}\left(V_{j}\right)$, the third equality follows from the independence of $V_{1}, V_{2}, \ldots, V_{n}$, and, in the last equation, $U:=V_{1} / s=$ $V_{1} / m(t)$ is a random variable uniformly distributed on the unit interval $[0,1]$.

According to (6), we obtain

$$
\begin{aligned}
& \mathrm{E}\left(\exp \left\{-\mu m^{-1}(s) N^{*}(s)+\mu \sum_{j=1}^{N^{*}(s)} T_{j}\right\}\right) \\
& =\mathrm{E}\left(\mathrm{E}\left(\exp \left\{-\mu m^{-1}(s) N^{*}(s)+\mu \sum_{j=1}^{N^{*}(s)} T_{j}\right\} \mid N^{*}(s)\right)\right) \\
& =\sum_{n=0}^{\infty} \mathrm{E}\left(\exp \left\{-\mu m^{-1}(s) n\right\} \exp \left\{\mu \sum_{j=1}^{n} T_{j}\right\} \mid N^{*}(s)=n\right) \mathrm{P}\left(N^{*}(s)=n\right) \\
& =\sum_{n=0}^{\infty} \exp \left\{-\mu m^{-1}(s) n\right\} \mathrm{E}\left(\exp \left\{\mu m^{-1}(s U)\right\}\right)^{n} \frac{s^{n}}{n !} \mathrm{e}^{-s} \\
& =\mathrm{e}^{-s} \sum_{n=0}^{\infty}\left[\exp \left\{-\mu m^{-1}(s)\right\} \mathrm{E}\left(\exp \left\{\mu m^{-1}(s U)\right\}\right) s\right]^{n} \frac{1}{n !} \\
& =\mathrm{e}^{-s} \exp \left\{\exp \left\{-\mu m^{-1}(s)\right\} \mathrm{E}\left(\exp \left\{\mu m^{-1}(s U)\right\}\right) s\right\}
\end{aligned}
$$

Observe that

$$
\mathrm{E}\left(\exp \left\{\mu m^{-1}(m(t) U)\right\}\right)=\int_{0}^{1} \exp \left\{\mu m^{-1}(m(t) x)\right\} \mathrm{d} x .
$$

If we here let $m^{-1}(m(t) x)=u$, then $x=(1 / m(t)) m(u)$ and

$$
\frac{\mathrm{d} x}{\mathrm{~d} u}=\frac{1}{m(t)} m^{\prime}(u)=\frac{1}{m(t)} \lambda(u) .
$$

Thus, we have

$$
\mathrm{E}\left(\exp \left\{\mu m^{-1}(m(t) U)\right\}\right)=\frac{\int_{0}^{t} \mathrm{e}^{\mu u} \lambda(u) \mathrm{d} u}{m(t)} .
$$

Combining (4), (7), and (8) yields

$$
\mathrm{P}(Y>t)=\bar{F}_{0}(t) \mathrm{e}^{-m(t)} \exp \left\{\mathrm{e}^{-\mu t} \int_{0}^{t} \mathrm{e}^{\mu x} \lambda(x) \mathrm{d} x\right\} .
$$


From (9), we have

$$
\ln \mathrm{P}(Y>t)=-\int_{0}^{t} r_{0}(x) \mathrm{d} x-m(t)+\mathrm{e}^{-\mu t} \int_{0}^{t} \mathrm{e}^{\mu x} \lambda(x) \mathrm{d} x .
$$

The compound failure rate function, $r(t)$, is thus given by

$$
\begin{aligned}
r(t) & =-\frac{\mathrm{d}}{\mathrm{d} t} \ln \mathrm{P}(Y>t) \\
& =-\frac{\mathrm{d}}{\mathrm{d} t}\left\{-\int_{0}^{t} r_{0}(x) \mathrm{d} x-m(t)+\mathrm{e}^{-\mu t} \int_{0}^{t} \mathrm{e}^{\mu x} \lambda(x) \mathrm{d} x\right\} \\
& =r_{0}(t)+\mu \mathrm{e}^{-\mu t} \int_{0}^{t} \mathrm{e}^{\mu x} \lambda(x) \mathrm{d} x .
\end{aligned}
$$

The following corollary gives a simpler expression for the compound failure rate function when the shock process is given by a homogeneous Poisson process with a constant intensity $\lambda>0$.

Corollary 1. If the shock process, $\{N(t): t \geq 0\}$, is a Poisson process with a constant intensity $\lambda>0$, then the compound failure rate function $r(t)$ is given by

$$
r(t)=r_{0}(t)+\lambda\left(1-\mathrm{e}^{-\mu t}\right) .
$$

\section{Various shapes of the compound failure rate function}

In this section we will discuss the limiting behavior of the compound failure rate function, and show that it can take many different shapes, including IFR (increasing failure rate), upside-down unimodal, right-side-up unimodal, bathtub, and even CFR (constant failure rate) and DFR (decreasing failure rate). We will use $\delta\left(r_{0}(\cdot), \lambda(\cdot), \mu\right)$ to denote the system with the baseline failure rate function $r_{0}(t)$ operated under the environmental condition $(\lambda(\cdot), \mu)$. Furthermore, in order to emphasize the dependence on $(\lambda(\cdot), \mu)$, we respectively denote the lifetime, compound failure rate function, and reliability function of the system $\delta\left(r_{0}(\cdot), \lambda(\cdot), \mu\right)$ as $Y_{(\lambda(\cdot), \mu)}, r(t ; \lambda(\cdot), \mu)$, and $R(t ; \lambda(\cdot), \mu)$ when it is necessary. In the discussions below, without loss of generality we assume that $\lambda(t)$ is greater than 0 and is differentiable for all $t \geq 0$.

Theorem 2. If $\lim _{t \rightarrow \infty} r_{0}(t)=: r_{0}(\infty) \leq \infty$ and $\lim _{t \rightarrow \infty} \lambda(t)=: \lambda(\infty) \leq \infty$ exist, then

$$
\begin{aligned}
r(\infty ; \lambda(\cdot), \mu): & =\lim _{t \rightarrow \infty} r(t ; \lambda(\cdot), \mu) \\
& = \begin{cases}r_{0}(\infty) & \text { if } \int_{0}^{\infty} \mathrm{e}^{\mu x} \lambda(x) \mathrm{d} x<\infty, \\
r_{0}(\infty)+\lambda(\infty) & \text { if } \int_{0}^{\infty} \mathrm{e}^{\mu x} \lambda(x) \mathrm{d} x=\infty\end{cases}
\end{aligned}
$$

Proof. From (2), the result is obvious for $\int_{0}^{\infty} \mathrm{e}^{\mu x} \lambda(x) \mathrm{d} x<\infty$. For $\int_{0}^{\infty} \mathrm{e}^{\mu x} \lambda(x) \mathrm{d} x=\infty$, application of l'Hôpital's rule gives the result immediately.

Corollary 2. Under the conditions of Theorem 2,

$$
r(\infty ; \lambda(\cdot), \mu)= \begin{cases}r_{0}(\infty) & \text { if } \mathrm{E}\left(T_{1}\right)=\infty \\ r_{0}(\infty)+\lambda(\infty) & \text { if } \mathrm{E}\left(T_{1}\right)<\infty\end{cases}
$$


Proof. We claim that $\mathrm{E}\left(T_{1}\right)=\infty$ implies $\lambda(\infty)=0$. This can be proved by contradiction. Suppose that the contrary result holds, and let $2 c:=\lambda(\infty)>0$. Then there exists a $t_{0}>0$ such that

$$
\lambda(x)>c \text { for } x \geq t_{0} .
$$

This implies that, for any $t \geq t_{0}$,

$$
\int_{0}^{t} \lambda(x) \mathrm{d} x \geq \int_{t_{0}}^{t} \lambda(x) \mathrm{d} x \geq c\left(t-t_{0}\right)
$$

and, consequently,

$$
\begin{aligned}
\mathrm{E}\left(T_{1}\right) & =\int_{0}^{\infty} \exp \left\{-\int_{0}^{t} \lambda(x) \mathrm{d} x\right\} \mathrm{d} t \\
& =\int_{0}^{t_{0}} \exp \left\{-\int_{0}^{t} \lambda(x) \mathrm{d} x\right\} \mathrm{d} t+\int_{t_{0}}^{\infty} \exp \left\{-\int_{0}^{t} \lambda(x) \mathrm{d} x\right\} \mathrm{d} t \\
& <\int_{0}^{t_{0}} \exp \left\{-\int_{0}^{t} \lambda(x) \mathrm{d} x\right\} \mathrm{d} t+\int_{t_{0}}^{\infty} \mathrm{e}^{-c\left(t-t_{0}\right)} \mathrm{d} t \\
& =\int_{0}^{t_{0}} \exp \left\{-\int_{0}^{t} \lambda(x) \mathrm{d} x\right\} \mathrm{d} t+\frac{1}{c}<\infty
\end{aligned}
$$

which is a contradiction. Therefore, it must be true that $\lambda(\infty)=0$. Notice, by Theorem 2 , that if $\mathrm{E}\left(T_{1}\right)=\infty$ then no matter whether $\int_{0}^{\infty} \mathrm{e}^{\mu x} \lambda(x) \mathrm{d} x<\infty$ or $\int_{0}^{\infty} \mathrm{e}^{\mu x} \lambda(x) \mathrm{d} x=\infty$, it is always true that $r(\infty ; \lambda(\cdot), \mu)=r_{0}(\infty)$, since, from our claim, $\lambda(\infty)=0$.

Now suppose that $\mathrm{E}\left(T_{1}\right)<\infty$. In this case we have $\int_{0}^{\infty} \lambda(x) \mathrm{d} x=\infty$, since

$$
\mathrm{E}\left(T_{1}\right)=\int_{0}^{\infty} \exp \left\{-\int_{0}^{t} \lambda(x) \mathrm{d} x\right\} \mathrm{d} t<\infty
$$

implies that $\exp \left\{-\int_{0}^{\infty} \lambda(x) \mathrm{d} x\right\}=0$. Hence,

$$
\int_{0}^{\infty} \mathrm{e}^{\mu x} \lambda(x) \mathrm{d} x \geq \int_{0}^{\infty} \lambda(x) \mathrm{d} x=\infty
$$

and, consequently, $r(\infty ; \lambda(\cdot), \mu)=r_{0}(\infty)+\lambda(\infty)$ by Theorem 2 .

Remark 1. It is tempting to try to show that $\mathrm{E}\left(T_{1}\right)<\infty$ implies $\lambda(\infty)>0$. However, this is not true. Indeed, let us assume $T_{1}$ to have a log-normal distribution. It is known that the failure rate function of a log-normal distribution has an upside-down bathtub shape and that its limit decreases to 0 as $t$ tends to $\infty$.

Remark 2. Suppose that $\int_{0}^{\infty} \mathrm{e}^{\mu x} \lambda(x) \mathrm{d} x<\infty$; then

$$
\int_{0}^{\infty} \lambda(x) \mathrm{d} x<\int_{0}^{\infty} \mathrm{e}^{\mu x} \lambda(x) \mathrm{d} x<\infty .
$$

Furthermore, $\mathrm{E}\left(T_{1}\right)=\infty$. However, the reverse implication does not hold, as demonstrated by the following counterexample. Let $\lambda(x)=1 /(1+x), x \geq 0$. It is straightforward to see that $\exp \left\{-\int_{0}^{t} \lambda(x) \mathrm{d} x\right\}=1 /(1+t)$ and, hence,

$$
\mathrm{E}\left(T_{1}\right)=\int_{0}^{\infty} \exp \left\{-\int_{0}^{t} \lambda(x) \mathrm{d} x\right\} \mathrm{d} t=\infty .
$$


However, on the other hand it is easy to verify that

$$
\int_{0}^{\infty} \mathrm{e}^{\mu x} \lambda(x) \mathrm{d} x=\int_{0}^{\infty} \frac{\mathrm{e}^{\mu x}}{1+x} \mathrm{~d} x=\infty .
$$

Remark 3. The same example also shows that

$$
\int_{0}^{\infty} \mathrm{e}^{\mu x} \lambda(x) \mathrm{d} x=\infty \quad \Longrightarrow \quad \mathrm{E}\left(T_{1}\right)<\infty .
$$

Intuitively, Corollary 2 tells us that when $\mathrm{E}\left(T_{1}\right)=\infty$, that is, the first arrival time of the shock process is extremely long, then only very few shocks, if any, can occur and, thus, the shock process does not affect the limit of the compound failure rate as $t$ tends to $\infty$.

Conversely, if $\mathrm{E}\left(T_{1}\right)<\infty$ then $r(\infty ; \lambda(\cdot), \mu)$ is affected by the shock process as indicated in Corollary 2. It is surprising that the dependence of $r(\infty ; \lambda(\cdot), \mu)$ on the shock process is expressed only through $\lambda(\infty)$. Let us look at the case of a stationary Poisson shock process with $\lambda(t)=\lambda$ for any $t \geq 0$. From (9) we see that the contribution of the shock process to the survival function of $Y$ is to add a series component with survival function

$$
\bar{G}(t):=\exp \left\{-\lambda\left(t-\frac{\mathrm{e}^{\mu t}-1}{\mu \mathrm{e}^{\mu t}}\right)\right\} .
$$

Note that the exponent in $\bar{G}(t)$ is equivalent to $-\lambda\left(t-\mu^{-1}\right)$ as $t \rightarrow \infty$. That is, for large $t$ the effect of the shock model on the survival function of $Y$ amounts to the addition in series to the original system of a component with a two-parameter exponential distribution. This twoparameter exponential distribution has a threshold $1 / \mu$ and the limit of its failure rate function as $t \rightarrow \infty$ does not depend on the threshold. Therefore, in the limiting case $\mu$ does not enter the expression for $r(\infty ; \lambda(\cdot), \mu)$. This situation is not uncommon. In fact, many widely applied lifetime distributions, such as Weibull, gamma, log-normal, and log-logistic distributions, have two parameters but are such that the limits of their failure rate functions as $t \rightarrow \infty$ either depend on only one of the two parameters or do not depend on either of them.

Lifetime distributions are often classified into different classes such as IFR, CFR, and DFR. To study the behavior of the compound failure rate function (2), we assume that $\lambda(t)$ is differentiable with $\lambda(0)<\infty$ and then rewrite (2) as

$$
\begin{aligned}
r(t ; \lambda(\cdot), \mu) & =r_{0}(t)+\mu \mathrm{e}^{-\mu t}\left(\left[\frac{1}{\mu} \mathrm{e}^{\mu x} \lambda(x)\right]_{0}^{t}-\frac{1}{\mu} \int_{0}^{t} \mathrm{e}^{\mu x} \lambda^{\prime}(x) \mathrm{d} x\right) \\
& =r_{0}(t)+\lambda(t)-\lambda(0) \mathrm{e}^{-\mu t}-\int_{0}^{t} \mathrm{e}^{-\mu(t-x)} \lambda^{\prime}(x) \mathrm{d} x .
\end{aligned}
$$

Also, note that the derivative of $r(t ; \lambda(\cdot), \mu)$ with respect to $t$ is

$$
r^{\prime}(t ; \lambda(\cdot), \mu)=r_{0}^{\prime}(t)+\mu \mathrm{e}^{-\mu t}\left(\lambda(0)+\int_{0}^{t} \mathrm{e}^{\mu x} \lambda^{\prime}(x) \mathrm{d} x\right) .
$$

The following examples demonstrate that the compound failure rate function $r(t ; \lambda(\cdot), \mu)$ can take various different shapes.

Example 1. (IFR shape.) Suppose that $r_{0}(t)=r_{0}$ for all $t \geq 0$ (i.e. the lifetime distribution is CFR). Then in this case $r_{0}^{\prime}(t)=0$ and the lifetime distribution of $Y$ is IFR if $\lambda^{\prime}(t) \geq 0$. 
This implies that, even though the lifetime distribution of $X$ is exponential (i.e. the system never deteriorates), preventive maintenance may have to be considered when it is operated in a random environment in which the intensity function $\lambda(t)$ is nondecreasing in $t$.

Specifically, let the baseline failure rate function be $r_{0}(t)=1, t \geq 0$, and let the environmental condition be given by $(\lambda(t), \mu)=\left(2-\mathrm{e}^{-t}, 1\right)$. In this case, the compound failure rate function in (2) is given by

$$
r(t ; \lambda(\cdot), 1)=3-t \mathrm{e}^{-t}-2 \mathrm{e}^{-t}, \quad t \geq 0,
$$

and $r^{\prime}(t ; \lambda(\cdot), 1)=(t+1) \mathrm{e}^{-t}>0, t \geq 0$.

Example 2. (Upside-down unimodal shape.) Suppose that $r_{0}(t)=r_{0}$ for all $t \geq 0$, and let $\lambda(0)>0$. In this case, from (12),

$$
r^{\prime}(t ; \lambda(\cdot), \mu)=\mu \mathrm{e}^{-\mu t}\left(\lambda(0)+\int_{0}^{t} \mathrm{e}^{\mu x} \lambda^{\prime}(x) \mathrm{d} x\right) .
$$

We see that $r^{\prime}(0+; \lambda(\cdot), \mu)>0$ and, thus, the compound failure rate function $r(t ; \lambda(\cdot), \mu)$ is initially increasing. It is also easy to see that $r(0 ; \lambda(\cdot), \mu)=r_{0}$ and, by $(2)$, that $r(t ; \lambda(\cdot), \mu)>$ $r_{0}$ for all $t>0$, regardless of the shape of $\lambda(t)$.

Now let us assume that $\lambda^{\prime}(t)<0$ for all $t>0$ and that $\int_{0}^{\infty} \mathrm{e}^{\mu x} \lambda^{\prime}(x) \mathrm{d} x<-\lambda(0)$. Let $g(t):=\lambda(0)+\int_{0}^{t} \mathrm{e}^{\mu x} \lambda^{\prime}(x) \mathrm{d} x$. Then $r^{\prime}(t ; \lambda(\cdot), \mu)=\mu \mathrm{e}^{-\mu t} g(t)$ and $g^{\prime}(t)=\lambda^{\prime}(t) \mathrm{e}^{\mu t}<0$, for all $t \geq 0$. Thus, it follows from $g^{\prime}(t)<0$, for all $t>0, g(0)=\lambda(0)>0$, and

$$
g(\infty):=\lim _{t \rightarrow \infty} g(t)=\lambda(0)+\int_{0}^{\infty} \mathrm{e}^{\mu x} \lambda^{\prime}(x) \mathrm{d} x<0,
$$

that there exists a positive value $t^{*}$ such that $r^{\prime}(t ; \lambda(\cdot), \mu)>0$ for all $t<t^{*}$ and $r^{\prime}(t ; \lambda(\cdot), \mu)<$ 0 for all $t>t^{*}$. That is, $r(t ; \lambda(\cdot), \mu)$ has an upside-down unimodal shape with change point $t^{*}$.

In particular, suppose that $\lambda(t)=\mathrm{e}^{-\eta t}$ for all $t \geq 0$, with $\eta>\mu$. Then it is obvious that $\lambda^{\prime}(t)<0$ and that

$$
\int_{0}^{\infty} \mathrm{e}^{\mu x} \lambda^{\prime}(x) \mathrm{d} x=-\frac{\eta}{\eta-\mu}<-1=-\lambda(0) .
$$

Note that

$$
\lambda(0)+\int_{0}^{t} \mathrm{e}^{\mu x} \lambda^{\prime}(x) \mathrm{d} x=1+\frac{\eta}{\eta-\mu}\left[\mathrm{e}^{-(\eta-\mu) t}-1\right] .
$$

From this we can see that $r^{\prime}(t ; \lambda(\cdot), \mu)>0$ for all $t<-(1 /(\eta-\mu)) \ln (\mu / \eta)$ and that $r^{\prime}(t ; \lambda(\cdot), \mu)<0$ for all $t>-(1 /(\eta-\mu)) \ln (\mu / \eta)$. This implies that $r(t ; \lambda(\cdot), \mu)$ is strictly increasing for $t<-(1 /(\eta-\mu)) \ln (\mu / \eta)$ and strictly decreasing for $t>-(1 /(\eta-\mu)) \ln (\mu / \eta)$. Hence, $r(t ; \lambda(\cdot), \mu)$ has an upside-down unimodal shape with change point given by $t^{*}=$ $-(1 /(\eta-\mu)) \ln (\mu / \eta)$. Moreover, from the first case of $(10)$, we have $\lim _{t \rightarrow \infty} r(t ; \lambda(\cdot), \mu)=$ $r_{0}$.

Specifically, suppose that the baseline failure rate function is given by $r_{0}(t)=1$ for all $t \geq 0$ and that the environmental condition is given by $(\lambda(t), \mu)=\left(\mathrm{e}^{-3 t}, 1\right)$. In this case, the compound failure rate function is given by

$$
r(t ; \lambda(\cdot), 1)=1+\frac{1}{2} \mathrm{e}^{-t}-\frac{1}{2} \mathrm{e}^{-3 t}, \quad t \geq 0 .
$$

In this example, the compound failure rate function strictly increases for all $t<0.5493$ and strictly decreases for all $t>0.5493$; it thus has an upside-down unimodal shape with 
change point $t^{*}=0.5493$. Furthermore, $\lambda(t)$ satisfies the condition in the first case of (10), i.e. $\int_{0}^{\infty} \mathrm{e}^{\mu x} \lambda(x) \mathrm{d} x<\infty$, and, thus, the limit of the compound failure rate function is given by $r_{0}(\infty)=1$.

The above example is very interesting in the following sense. From (1), given the shock process $\{N(t): t \geq 0\}$, the conditional failure rate of the system is given by

$$
r_{0}(t)+\sum_{j=1}^{N(t)} \mu \mathbf{1}_{\left[T_{j}, \infty\right)}(t),
$$

which is a nondecreasing function of $t>0$. Furthermore, from (13) we can see that the occurrence of a shock affects the failure rate not only at the time of its occurrence, but also continuously and indefinitely starting from the time of its occurrence, regardless of the shape of $\lambda(t)$. Thus, intuitively it would seem that $r(t ; \lambda(\cdot), \mu)$ is always IFR. However, from the above discussion we see that the compound failure rate is not always IFR. In fact, it can take various different shapes, including even DFR shape, as the following examples demonstrate.

Example 3. (DFR shape.) Let $\lambda(t)=1$ for all $t \geq 0$ and let $\mu=1$. Then $r^{\prime}(t ; \lambda(\cdot), \mu)=$ $r_{0}^{\prime}(t)+\mathrm{e}^{-t}$. If, furthermore, $r_{0}(t)=2 \mathrm{e}^{-t}$, then $r^{\prime}(t ; \lambda(\cdot), \mu)=-\mathrm{e}^{-t}<0$ and, so, the compound failure rate is DFR.

Let us show that $r(t ; \lambda(\cdot), \mu)$ can have a right-side-up unimodal shape.

Example 4. (Right-side-up unimodal shape.) Let $\lambda(t)=\lambda$ for all $t \geq 0$ and let $r_{0}(t)=\mathrm{e}^{-\xi t}$ for all $t \geq 0$, with $\xi>\mu \lambda$ and $\xi>\mu$. Then, from Corollary 1, the compound failure rate function is given by

$$
r(t ; \lambda(\cdot), \mu)=r_{0}(t)+\lambda\left(1-\mathrm{e}^{-\mu t}\right)=\mathrm{e}^{-\xi t}+\lambda-\lambda \mathrm{e}^{-\mu t},
$$

and its derivative with respect to $t$ is given by

$$
r^{\prime}(t ; \lambda(\cdot), \mu)=-\xi \mathrm{e}^{-\xi t}+\lambda \mu \mathrm{e}^{-\mu t}
$$

It is thus easy to see that

$$
r^{\prime}(t ; \lambda(\cdot), \mu) \begin{cases}<0 & \text { if } 0<t<t^{*}, \\ >0 & \text { if } t>t^{*}\end{cases}
$$

where $t^{*}=-(1 /(\xi-\mu)) \ln (\lambda \mu / \xi)$. That is, in this example $r(t ; \lambda(\cdot), \mu)$ has a right-side-up unimodal shape with change point $t^{*}$.

In particular, if we choose $\mu=0.5, \lambda=1.0$, and $\xi=3.0$, then the graph of the compound failure rate function is as plotted in Figure 1. As is shown there, the compound failure rate function has a right-side-up unimodal shape with change point $t^{*}=0.7167$.

The following example shows that $r(t ; \lambda(\cdot), \mu)$ can be bathtub shaped.

Example 5. (Bathtub shape.) Let $\lambda(t)=\lambda$ for all $t \geq 0$ and let

$$
r_{0}(t)= \begin{cases}\mathrm{e}^{-t}+1 & \text { if } 0 \leq t \leq t_{1} \\ \mathrm{e}^{-t_{1}}-\mu^{-1} \mathrm{e}^{-t_{1}}\left[1-\mathrm{e}^{-\mu\left(t-t_{1}\right)}\right]+1 & \text { if } t_{1} \leq t \leq t_{2} \\ 0.1\left(t-t_{2}\right)^{2}+1 & \text { if } t_{2} \leq t\end{cases}
$$




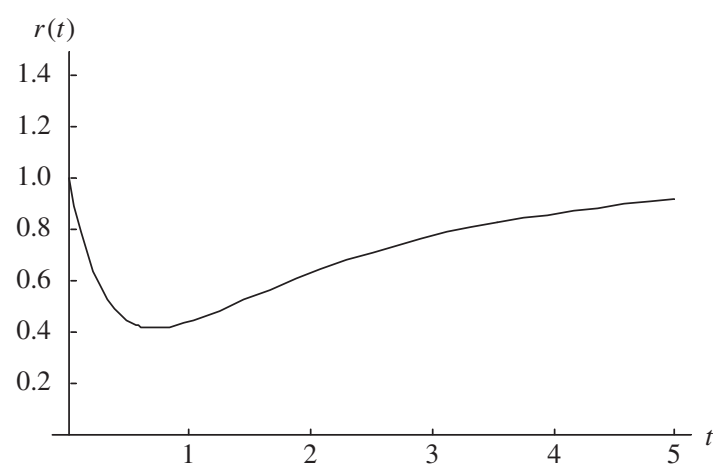

FIGURE 1: The compound failure rate function in the right-side-up unimodal case.

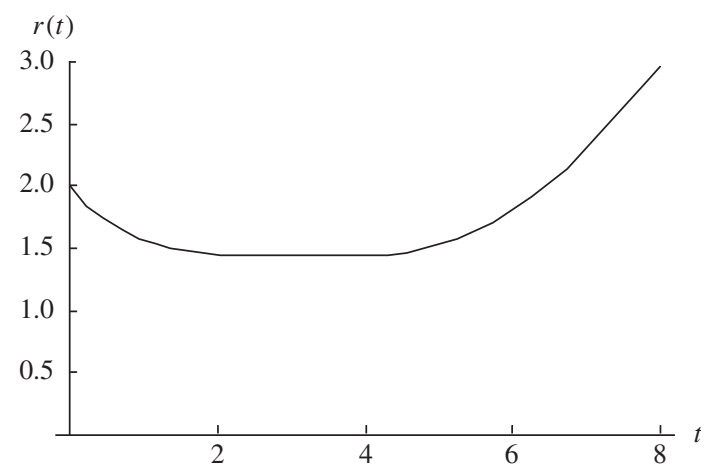

FIGURE 2: Compound failure rate function in the bathtub-shape case.

where $\mu<1, \lambda<1, t_{1}=-(1-\mu)^{-1} \ln (\lambda \mu)$, and $t_{2}=t_{1}-\mu^{-1} \ln (1-\mu)$. Then $r(t ; \lambda(\cdot), \mu)$ has a bathtub shape with two different change points, $t_{1}$ and $t_{2}, t_{1}<t_{2}$, and $r(t ; \lambda(\cdot), \mu)$ is a constant on the interval $\left[t_{1}, t_{2}\right]$.

In particular, if we choose $\lambda=0.5$ and $\mu=0.5$, then the graph of the compound failure rate function is as plotted in Figure 2. As is shown there, the compound failure rate function is bathtub shaped with change points $t_{1}=4 \ln 2$ and $t_{2}=6 \ln 2$.

Finally, we show that $r(t ; \lambda(\cdot), \mu)$ can be a constant function.

Example 6. (CFR.) It can be easily verified using (2) and (12) that $r(t ; \lambda(\cdot), \mu)$ is a constant function if and only if the following two conditions are satisfied:

(a) $\lambda(0)=-\frac{1}{\mu} r_{0}^{\prime}(0) \geq 0$,

(b) $\lambda^{\prime}(x)=-\mu^{-1} \mathrm{e}^{-\mu x} \frac{\mathrm{d}}{\mathrm{d} x}\left(\mathrm{e}^{\mu x} r_{0}^{\prime}(x)\right)$.

Now, if we choose $r_{0}(t)=\mathrm{e}^{-t}$ for all $t \geq 0$ and let $\mu>0$ be arbitrary, then we will obtain

$$
\lambda(t)=1-\left(1-\mu^{-1}\right) \mathrm{e}^{-t}, \quad t \geq 0 .
$$

Direct computation based on (2) gives $r(t ; \lambda(\cdot), \mu)=1, t \geq 0$. 


\section{Dependence on the intensity function and the increment in failure rate}

To study the dependence of $r(t ; \lambda(\cdot), \mu)$ on $\lambda(\cdot)$ and $\mu$ we need the concept of failure rate ordering (see, e.g. Shaked and Shanthikumar (1994, p. 12)).

Definition 1. Let $Z_{1}$ and $Z_{2}$ be two nonnegative, continuous random variables with respective failure rate functions, $q_{1}(\cdot)$ and $q_{2}(\cdot)$, such that

$$
q_{1}(t) \geq q_{2}(t), \quad t \geq 0
$$

Then $Z_{1}$ is said to be smaller than $Z_{2}$ in the failure rate order, written $Z_{1} \leq_{\text {fr }} Z_{2}$.

Theorem 3. (I) Consider systems $\&\left(r_{0}(\cdot), \lambda_{1}(\cdot), \mu\right)$ and $\&\left(r_{0}(\cdot), \lambda_{2}(\cdot), \mu\right)$. If $\lambda_{1}(t) \geq \lambda_{2}(t)$ for all $t \geq 0$ then $Y_{\left(\lambda_{1}(\cdot), \mu\right)} \leq_{\text {fr }} Y_{\left(\lambda_{2}(\cdot), \mu\right)}$.

(II) Consider systems $s\left(r_{0}(\cdot), \lambda(\cdot), \mu_{1}\right)$ and $s\left(r_{0}(\cdot), \lambda(\cdot), \mu_{2}\right)$. If $\mu_{1} \geq \mu_{2}$ and $\lambda^{\prime}(t) \geq 0$ then $Y_{\left(\lambda(\cdot), \mu_{1}\right)} \leq_{\text {fr }} Y_{\left(\lambda(\cdot), \mu_{2}\right)}$.

Proof. From (2), it is easy to see that if $\lambda_{1}(t) \geq \lambda_{2}(t)$ for all $t>0$ then $r\left(t ; \lambda_{1}(\cdot), \mu\right) \geq$ $r\left(t ; \lambda_{2}(\cdot), \mu\right)$ for all $t>0$. That is, $Y_{\left(\lambda_{1}(\cdot), \mu\right)} \leq_{\mathrm{fr}} Y_{\left(\lambda_{2}(\cdot), \mu\right)}$.

From (11), the compound failure rate function in (2) is given by

$$
r(t ; \lambda(\cdot), \mu)=r_{0}(t)+\lambda(t)-\lambda(0) \mathrm{e}^{-\mu t}-\int_{0}^{t} \mathrm{e}^{-\mu(t-x)} \lambda^{\prime}(x) \mathrm{d} x .
$$

Hence,

$$
\frac{\partial r(t ; \lambda(\cdot), \mu)}{\partial \mu}=\lambda(0) t \mathrm{e}^{-\mu t}+\int_{0}^{t}(t-x) \exp \{-\mu(t-x)\} \lambda^{\prime}(x) \mathrm{d} x .
$$

Therefore, if $\lambda^{\prime}(t) \geq 0$ for all $t \geq 0$ then, for any $t>0, r(t ; \lambda(\cdot), \mu)$ is strictly increasing in $\mu>0$.

It is worthy of note that if the condition $\lambda^{\prime}(t) \geq 0$ is violated in Theorem 3 , then the compound failure rate function may not be strictly increasing in $\mu$ for all $t>0$, as indicated by the following example.

Example 7. Suppose that the baseline failure rate function is given by $r_{0}(t)=1$ for all $t \geq 0$. For this system, we consider two different environmental conditions, $\left(\lambda(t), \mu_{1}\right)=\left(\mathrm{e}^{-3 t}, 1\right)$ and $\left(\lambda(t), \mu_{2}\right)=\left(\mathrm{e}^{-3 t}, 2\right)$. The corresponding compound failure rate functions are respectively given by

$$
r_{1}(t):=r(t ; \lambda(\cdot), 1)=1+\frac{1}{2} \mathrm{e}^{-t}-\frac{1}{2} \mathrm{e}^{-3 t}, \quad t \geq 0,
$$

and

$$
r_{2}(t):=r(t ; \lambda(\cdot), 2)=1+2 \mathrm{e}^{-2 t}-2 \mathrm{e}^{-3 t}, \quad t \geq 0 .
$$

It can be shown that $r_{2}(t)>r_{1}(t)$ for $t \in(0, \ln 3)$, that $r_{2}(t)<r_{1}(t)$ for $t>\ln 3$, and that $r_{2}(t)=r_{1}(t)$ for $t=0$ or $t=\ln 3$. The graphs of these two compound failure rate functions are plotted in Figure 3. 


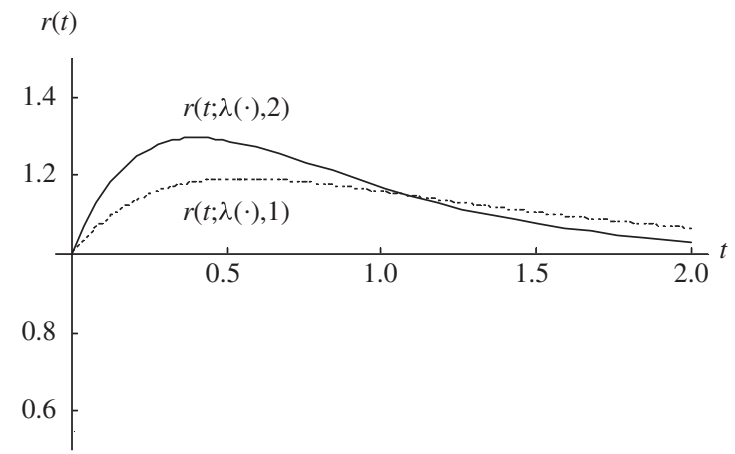

FIGURE 3: The graphs of two compound failure rate functions.

\section{Concluding remarks}

When a system is used under varying environmental conditions, the environmental factors should be incorporated into the study of the system performance.

In this paper, we have studied the performance of a system which is operated in a random environment governed by a Poisson shock process. From the results for the compound failure rate function, it can be seen that the influence of the occurrence rate of the shock process is more significant than that of the amount of failure rate increment caused by each shock. Furthermore, we obtained some surprising results which could not have been predicted prior to analysis.

In future study, more general environmental factors, such as pressure, temperature, humidity, and so on, could be modeled via various stochastic processes to describe the operational characteristics of the real world. Also note that in much of the previous research work on statistical inference, only the parameters in the baseline failure rate function have received attention. Since the environmental factors are essential elements of the system performance, more attention should be paid to parameters describing environmental conditions in future research.

\section{Acknowledgements}

The authors thank the referee and the Editor for helpful comments and careful readings of this paper, which have improved its presentation considerably.

\section{References}

Abdel-Hameed, M. S. and Proschan, F. (1973). Nonstationary shock models. Stoch. Process. Appl. 1, $383-404$. A-Hameed, M. S. and Proschan, F. (1975). Shock models with underlying birth process. J. Appl. Prob. 12, $18-28$. ARJAS, E. (1981). The failure and hazard process in multivariate reliability systems. Math. Operat. Res. 6, 551-562. ÇInlar, E. (1975). Introduction to Stochastic Processes. Prentice-Hall, Englewood Cliffs, NJ.

ÇInLAR, E. (1977). Shock and wear models and Markov additive processes. In The Theory and Applications of Reliability, Vol. 1, eds I. N. Shimi and C. P. Tsokos, Academic Press, New York, pp. 193-214.

ÇINLAR, E. (1984). Markov and semi-Markov models of deterioration. In Reliability Theory and Models, eds M. S. A-Hameed, E. Çinlar and J. Quinn, Academic Press, Orlando, FL, pp. 3-41.

ÇInlar, E. AND Özekici, S. (1987). Reliability of complex systems in random environments. Prob. Eng. Inf. Sci. 1, 97-115.

Çinlar, E., Shaked, M. and Shanthikumar, J. G. (1989). On lifetimes influenced by a common environment. Stoch. Process. Appl. 33, 347-359.

Esary, J. D. ANd Marshall, A. W. (1974). Families of components, and systems, exposed to a compound damage process. In Reliability and Biometry: Statistical Analysis of Lifelength (Proc. Conf., Florida State Univ., Tallahassee, FL, 1973), eds F. Proschan and R. J. Serfling, SIAM, Philadelphia, PA, pp. 31-46. 
Esary, J. D., Marshall, A. W. and Proschan, F. (1973). Shock models and wear processes. Ann. Prob. 1, 627-649. Feldman, R. M. (1976). Optimal replacement with semi-Markov shock models. J. Appl. Prob. 13, 108-117.

Feldman, R. M. (1977). Optimal replacement for systems governed by Markov additive shock processes. Ann. Prob. 5, 413-429.

GAVER, D. P. (1963). Random hazard in reliability problems. Technometrics 5, 211-226.

KebIR, Y. (1991). On hazard rate process. Naval Res. Logistics 38, 865-876.

Lemoine, A. J. And Wenocur, M. L. (1985). On failure modeling. Naval Res. Logistics 32, 497-508.

Lemoine, A. J. And Wenocur, M. L. (1986). A note on shot-noise and reliability modeling. Operat. Res. 34, $320-323$.

ÖZEKICI, S. (1995). Optimal maintenance policies in random environments. Europ. J. Operat. Res. 82, $283-294$.

ÖzeKICI, S. (1996). Complex systems in random environments. In Reliability and Maintenance of Complex Systems, ed. S. Özekici, Springer, Berlin, pp. 137-157.

Shaked, M. and Shanthikumar, J. G. (1989). Some replacement policies in a random environment. Prob. Eng. Inf. Sci. 3, 117-134.

Shaked, M. and Shanthikumar, J. G. (1994). Stochastic Orders and Their Applications. Academic Press, New York. SingPurwalla, N. D. (1995). Survival in dynamic environments. Statist. Sci. 10, 86-103. 